LBNL - 52156

\title{
On the influence of forests on the overall fate of semivolatile organic contaminants
}

\author{
Matthew MacLeod \\ Environmental Energy Technologies Division \\ Indoor Environment Department \\ Lawrence Berkeley National Laboratory \\ One Cyclotron Road \\ Berkeley, CA 94720
}

February 2003

This work was supported by the Natural Sciences and Engineering Research Council of Canada (NSERC) and by the US Environmental Protection Agency National Exposure Research Laboratory through Interagency Agreement \# DW-988-38190-01-0, carried out at Lawrence Berkeley National Laboratory through the US Department of Energy under Contract No. DE-AC03-76SF00098. 
Question 1. Is there a Forest Filter Effect?

Convincing experimental evidence exists that the presence of vegetation, especially forest canopies, enhances scavenging of semi-volatile organic chemicals (SVOCs) from the atmosphere and increases their depositional flux to the terrestrial surface relative to deposition to bare soil (Horstmann and McLachlan 1998, McLachlan and Horstmann 1998). Evidence from several modeling studies (McLachlan and Horstmann 1998, Wania and McLachlan 2001, Cousins and Mackay 2001, Severinsen and Jager 1998) indicates that gaseous deposition to vegetation is the most efficient scavenging pathway, and, due to interactions between gas-particle partitioning in the atmosphere and resistance to diffusion through the cuticle of plants, that pathway is available for SVOCs with Log Koa between 7 and 11 and Log Kaw > -6.

However, for enhanced scavenging by vegetation to influence the overall regional and global fate of SVOCs, it must represent a process that is competitive with the other fate and transport processes in the ecological system under consideration. The key question in evaluating the importance of the "Forest Filter Effect" on the regional and global fate of SVOCs is: Does scavenging from the atmosphere by vegetation have a significant influence on the inter-media partitioning, long-range transport and/or environmental persistence of SVOCs? Based on evidence presented below from the CoZMo-POP model and supported by results from other models that together represent our current state of knowledge, the Forest Filter Effect does not exert a controlling influence over regional or global contaminant fate and transport.

Question 2. Model evidence of the dominance of other fate processes over the Forest Filter Effect 
In a regional environmental assessment, we are interested in whether SVOCs emitted in, or carried into a forested region are likely to be scavenged from the atmosphere and deposited to soils, thereby reducing their atmospheric transport and likely increasing their overall regional environmental persistence. For non-degrading SVOCs, this amounts to a competition between the process of advection through the region in the atmosphere and transfer to forests followed by deposition to soils.

As reported by Wania and McLachlan (2001), when advection is ignored transfer along the air-tovegetation-to-soil pathway strongly influences the atmospheric concentration of SVOCs calculated with the CoZMo-POP model. At the peak of efficiency of this process, for emissions to the atmosphere of a non-reacting hypothetical chemical with $\log \mathrm{Koa}=9.5, \log \mathrm{Kaw}=-2.5$ and $\log$ Kow $=7.0$, modeled contaminant concentrations in the air are decreased by a factor of approximately 5 relative to a scenario without vegetation. However, because the influence of air advection through the region is ignored, this factor of 5 reduction in air concentration clearly represents an upper bound on the possible influence of forest scavenging on atmospheric concentrations of non-degrading SVOCs.

Figure 1 shows results from the CoZMo-POP model for a non-degrading hypothetical chemical with partitioning properties described above emitted to the atmosphere for two scenarios that include advection in air - one with a forest canopy compartment covering $50 \%$ of the terrestrial surface, and one excluding the forest compartment. All other model parameters are identical to those used by Wania and McLachlan (2001). Figure 1 can be compared to their Figure 4, which neglects advection. The atmospheric residence time used in these calculations is 2 days, as recommended by Wania et al. (2000) for the $100000 \mathrm{~km}^{2}$ default regional environment of the CoZMo-POP model. 
[FIGURE 1]

As shown in Figure 1, advection in air is the dominant fate process that determines the overall level of regional contamination in the system. Contaminant concentrations in the atmosphere are reduced by the presence of vegetation by a factor of only 1.2. Similarly, the efficiency of chemical transport through the region, an indicator of long-range transport potential, is reduced by a factor of 1.2. The total rate of deposition from the atmosphere to the terrestrial surface is significantly increased in the scenario including vegetation, but this is partially balanced by a higher rate of re-volatilization, consistent with near-equilibrium partitioning between the atmosphere and the vegetation compartment and efficient bi-directional diffusive exchange. These results are broadly consistent with the earlier model analysis by Severinsen and Jager (1998) and results from the BETR North America model (MacLeod and Mackay 2002), which includes a vegetation compartment based on the work of Cousins and Mackay (2001). The reduction in atmospheric concentrations attributable to the Forest Filter Effect is believed to be small relative to overall uncertainties for a model of this type.

On the global scale the atmosphere can be considered to be a closed system (there is no advection of air in or out) and the Forest Filter Effect might be expected to play a more important role than on regional scales. Of the earth's land area $23 \%$ is forested, corresponding to $6.5 \%$ of its total area (http://www.globalforestwatch.org/english/about/faqs.htm). The oceans cover $70 \%$ of the earth's surface. A simple single-region global model parameterization of the CoZMo-POP model based on these data yielded a factor of 1.35 reduction in atmospheric concentrations for a scenario including forests compared to one without forests for the hypothetical non-reacting chemical with $\log \mathrm{Koa}=9.5, \log \mathrm{Kaw}=-2.5$ and $\log \mathrm{Kow}=7.0$ emitted to air. The dominant fate and transport processes in these scenarios were accumulation in ocean water, sediment-water exchange in the oceans and burial in oceanic sediments. As on the regional scale, the influence of 
the Forest Filter Effect on global-scale atmospheric concentrations is believed to be small relative to total uncertainties in the model calculation.

In summary, current models and experimental evidence indicate that there is a Forest Filter Effect, however, it does not exert a controlling influence on the regional and/or global-scale longrange transport potential and overall environmental persistence of semi-volatile organic chemicals. This conclusion is based on models derived from very few experimental studies of air-vegetation-soil exchange of SVOCs. Additional experiments under controlled laboratory conditions and in the field are required to improve our understanding of this process and its influence on the environmental behavior of contaminants. The present discussion ignores contaminant degradation in all media. The rates of degrading reactions of SVOCs incorporated in vegetation, or sorbed to the vegetation surface could be significantly different from those in the atmosphere or in soils, and thus influence contaminant dynamics. Local-scale effects of vegetation may also be important, for example in determining the relative rates of deposition to agricultural and forest soils within a region, which would influence human exposure to environmental contaminants through agricultural foods.

\section{Acknowledgments}

This work was supported by the Natural Sciences and Engineering Research Council of Canada (NSERC) and by the US Environmental Protection Agency National Exposure Research Laboratory through Interagency Agreement \# DW-988-38190-01-0, carried out at Lawrence Berkeley National Laboratory through the US Department of Energy under Contract No. DE-AC03-76SF00098. 


\section{References}

Cousins IT, Mackay D (2001) Strategies for including vegetation compartments in multimedia models. Chemosphere 44: 643-654

Horstmann M, McLachlan MS (1998) Atmospheric deposition of semivolatile organic compounds to two forest canopies. Atmospheric Environment 32: 1799-1809

MacLeod M, Mackay D (2002) Modeling transport and deposition of contaminants to ecosystems of concern: A case study for the Laurentian Great Lakes. Environmental Pollution (in review)

McLachlan M, Horstmann M (1998) Forests as filters of airborne organic pollutants: A model. Environmental Science and Technology 32: 413-420

Severinsen M, Jager T (1998) Modelling the influence of terrestrial vegetation on the environmental fate of xenobiotics. Chemosphere 37: 41-62

Wania F, Persson J, Di Guardo A, McLachlan MS (2000) CoZMo-POP. A fugacity-based multicompartmental mass balance model of the fate of persistent organic pollutants in the coastal zone. WECC Report 1/2000, Toronto, Canada. 26 pages. www.scar.utoronto.ca/ wania

Wania F, McLachlan M (2001) Estimating the influence of forests on the overall fate of semivolatile organic compounds using a multimedia fate model. Environmental Science and Technology 35: 582-590 
Figure 1. Cumulative fluxes of a chemical with $\log \mathrm{Kaw}=-2.5, \log \mathrm{Koa}=9.5$ and $\log \mathrm{Kow}=$ 7.0 in units of percentage of total cumulative emission over a 50 year simulation, assuming a zero background concentration and an atmospheric residence time of 2 days in a $100000 \mathrm{~km}^{2}$ region. Bold font is for simulation with agricultural soil only; regular font is for simulation assuming a mixed forest covers half of the terrestrial surface. 